\title{
Description and characterization of a novel live-attenuated tri-segmented Machupo virus in Guinea pigs
}

\author{
Amélie D. Zaza ${ }^{1,2^{*}}$, Cécile H. Herbreteau ${ }^{1}$ and Christophe N. Peyrefitte ${ }^{2,3}$
}

\begin{abstract}
Background: Machupo virus (MACV) is a member of the Mammarenavirus genus, Arenaviridae family and is the etiologic agent of Bolivian hemorrhagic fever, which causes small outbreaks or sporadic cases. Several other arenaviruses in South America Junín virus (JUNV) in Argentina, Guanarito in Venezuela, Sabiá in Brazil and Chapare in Bolivia, also are responsible for human hemorrhagic fevers. Among these arenaviruses, JUNV caused thousands of human cases until 1991, when the live attenuated Candid \#1 vaccine, was used. Other than Candid \#1 vaccine, few other therapeutic or prophylactic treatments exist. Therefore, new strategies for production of safe countermeasures with broad spectrum activity are needed.

Findings: We tested a tri-segmented MACV, a potential vaccine candidate with several mutations, (r3MACV). In cell culture, r3MACV showed a 2 -log reduction in infectious virus particle production and the MACV inhibition of INF-1 $\beta$ was removed from the construct and produced by infected cells. Furthermore, in an animal experiment, r3MACV was able to protect $50 \%$ of guinea pigs from a simultaneous lethal JUNV challenge. Protected animals didn't display clinical symptoms nor were virus particles found in peripheral blood (day 14) or in organs (day 28 post-inoculation). The r3MACV provided a higher protection than the Candid \#1 vaccine.
\end{abstract}

Conclusions: The r3MACV provides a potential countermeasure against two South America arenaviruses responsible of human hemorrhagic fever.

Keywords: Vaccine candidate development, Therapeutic, Mammarenaviruses, Machupo virus, Junín virus, Candid \#1, Tri-segmented virus, Reverse genetic approaches

\section{Findings}

Machupo virus (MACV) belongs to the Mammarenavirus genus of the Arenaviridae family and is the etiologic agent of Bolivian Hemorrhagic Fever (BHF) [1], discovered in 1959. From 1962 to 1964, it caused 515 human cases, 114 of which were fatal [1]. Then, 40 years later, from 2006through 2008, 200 human cases were reported including 12 deaths [2]. Similarly, the 1950s, another arenavirus, Junín virus (JUNV), was responsible for cases of human hemorrhagic fever (HHF) in Argentina [3]. Since 1991, the use of the Candid \#1 vaccine, a live-attenuated vaccine against JUNV, decreased the case

\footnotetext{
* Correspondence: ameliezaza@gmail.com

${ }^{1}$ Fab'entech, 24 rue Jean Baldassini Bat B 69007, Lyon, France

${ }^{2} U n i t e ́$ de virologie, Institut de Recherche Biomédicale des Armées, 1 place

Valérie André, 91220 Brétigny-sur-Orge, France

Full list of author information is available at the end of the article
}

number [3]. Furthermore, three others mammarenaviruses have caused HHF outbreaks in South America and include Guanarito (GTOV), Chapare (CHAPV) and Sabiá (SABV) viruses [4-7]. Post-exposure passive transfer of human plasma-derived antibodies was used for humans and also non-human primates (NHPs) challenged by JUNV and MACV [3, 8]. However, this treatment induced a late-neurological syndrome [8]. Although Ribavirin reduced lethality in humans, administration at very early stage of infection was necessary [9] due to its side effects [3]. Therefore, alternative strategies with a broad spectrum activity are needed. Here, we report the use of the reverse genetic strategy to design a potential vaccine candidate targeting MACV and JUNV.

Mammarenaviruses are enveloped viruses with a single-stranded RNA genome composed of two segments [9]. Each segment encodes two ambisense genes, separated

(c) The Author(s). 2018 Open Access This article is distributed under the terms of the Creative Commons Attribution 4.0 International License (http://creativecommons.org/licenses/by/4.0/), which permits unrestricted use, distribution, and 
by a hairpin intergenic region (Fig. 1a). The L segment contains the genes of the small ring finger $\mathrm{Z}$ protein and of the RNA-dependent RNA polymerase $\mathrm{L}$ protein. In the $\mathrm{S}$ segment are found the genes of the viral nucleoprotein (NP) and of the glycoprotein complex (GPC). It was previously demonstrated that the bi-segmented genome of arenaviruses could be modified into a tri-segmented genome, by the duplication of the $S$ segment [10]. To select and maintain tri-segmented viruses, the NP gene was removed from one of the two $\mathrm{S}$ segments, and the GPC gene was removed from the second S segment, so that two genes of interest could be inserted into these empty loci (Fig. 1b). Prior studies demonstrated efficacy using this approach for Old World Lymphocytic choriomeningitis virus (LCMV) or the New World JUNV [10, 11]. They constructed a tri-segmented JUNV (r3JUNV) or LCMV (r3LCMV), the latter of which showed high attenuation vs WT and exhibited protection against LCMV challenge when used as a vaccine candidate in mice [10].

In these studies, we used the reverse genetic system applied to the MACV Carvallo strain genome. As illustrated in Fig. 1 we generated three $S$ segments, a wild-type one along with two genetically modified S segments, one harboring the CHAPV strain 810,419 GPC gene (acc \# EU260463.1) into the MACV GPC gene locus, and the second displaying GTOV strain INH-95551 GPC gene (acc \# AY129247.1) into the MACV NP gene locus. This virus was named r3MACV (Fig. 1b). Thirty three (33) amino acids were deleted from the C-termini of the two GPC sequences, required for production of infectious viral particles [12], and avoid any competition with the MACV wt GPC. Such a competition might have changed the virus cellular tropism or led to the loss of one of the two modified $\mathrm{S}$ segment. Additional modifications aimed to stimulate the adaptive immune response, by insertion of mutation (D380A in NP gene), shown to prevent the MACV NP IFN type-I inhibition [13]. Finally, glutamic acid was replaced by glycine at position166 (E166G) in the polymerase sequence. This mutation resulted in an 8 -fold decrease in polymerase activity (mini-genome assay, Additional file 1). MACV and JUNV are closely genetically related, and, indeed, Candid \#1, protected guinea pigs and primates from a lethal MACV challenge [14]. Since SABV and CHAPV are genomically closely related [6], r3MACVwas designed to target MACV, JUNV and each these additional South America arenaviruses responsible of HHF.

To replicate the pattern of ther3LCMV construct, reverse genetic systems were designed using synthesized Z, GPC, NP, NP D380A and L genes as well as S and L backbones (Eurofins Genomics, France) based on published sequences (GenBank acc \# AY619643 and AY619642). The $\mathrm{S}$ and $\mathrm{L}$ backbones contained all the non-coding regions of the related viral segment, and the genes loci were replaced by BsmBI (GPC and L loci), BbsI (NP locus) or SapI (Z locus) restriction sites (Fig. 1). These sites were designed to further eliminate all the non-viral sequences after the enzymatic restriction step. Restriction sites required to clone the genes into the backbones or the pCAGGs plasmids were either included during the gene synthesis, or added by PCR using primers with $5^{\prime}$ extensions using the PCR Extender system (5PRIME) or the Q5 High-Fidelity PCR Kit (New England Biolabs), following manufacturer's recommendations [10]. The E166G polymerase mutation was inserted into the expression plasmids using the Q5 site-directed mutagenesis kit (New England Biolabs). Amplified products were then purified using the QIAquick PCR Purification kit (Qiagen) and digested with the corresponding enzyme (BsmBI, BbsI or SapI, New England Biolabs). Restriction products were gel purified using agarose (Thermo Fisher) and the QIAquick Gel Extraction Kit (Qiagen) then ligated using the Quick Ligation Kit (New England Biolabs). Ligated products were transformed into the NEB $10-\beta$ or NEB 5- $\alpha$ F'Iq Competent E. coli (New England Biolabs). The bacterial clones were screened using the PCR (PCR Master Mix,
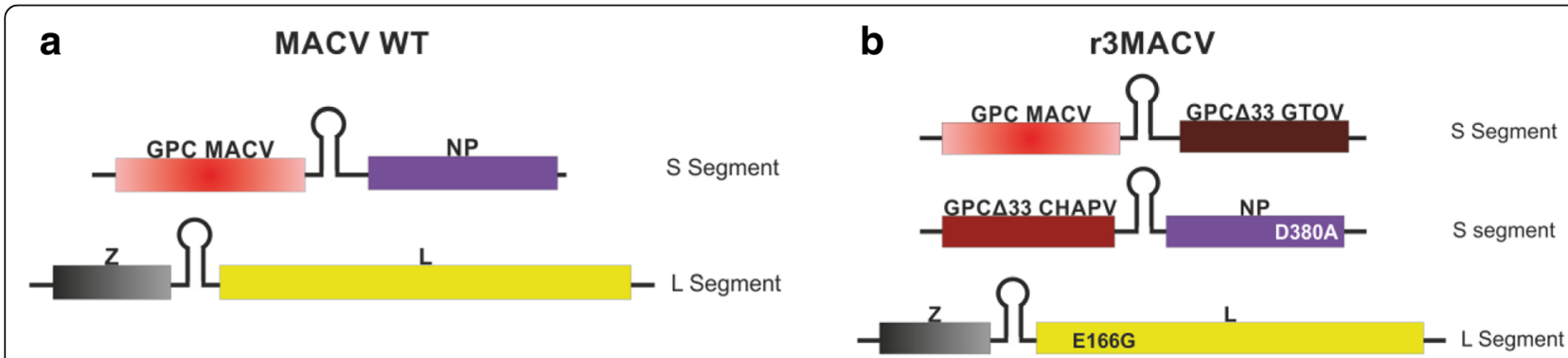

Fig. 1 Schematic representation of MACV wt and r3MACV genomic constructs. Arenaviruses have a bisegmented negative single-stranded RNA genome. a For the MACV wt, the $S$ segment encodes the viral glycoprotein precursor (GPC) and the nucleoprotein (NP), whereas $L$ segment encodes the viral polymerase (L) and the small RING finger protein Z. b For the r3MACV, one $S$ segment encodes the MACV GPC and the GTOV GPC $\triangle 33$, and the other encodes the CHAPV GPC $\triangle 33$ and the MACV NP, and the $L$ segment is unchanged than the wt genome. Mutations inserted in r3MACV genome were noted 
Thermo Fisher) following the manufacturer's instructions. The positive clones were grown in $50 \mathrm{ml} \mathrm{LB}$ broth (Thermo Fisher), then the plasmids were extracted using the Plasmid Plus Midi Kit (Qiagen). Two vectors, kindly provided by JC de la Torre, were used: the pCAGGs vector to express the viral proteins [15], and the pol I vector to produce the viral RNA segments [16]. The r3MACV genomic sequences are shown in Additional file 2.

After the construction and production of the MACV wt and r3MACV, using conditions previously described [11], viruses were concentrated and purified using an ultracentrifugation step $\left(28,000 \mathrm{rpm}\right.$ for $2 \mathrm{~h}$ at $4{ }^{\circ} \mathrm{C}$ in a Beckman sw32i rotor) on a sucrose (Life technologies) gradient (20 to 60\%). Pellets were resuspended into PBS (Life technologies). We then compared their replication and propagation in VERO cells at a multiplicity of infection (MOI) of 0.01 . We observed at 48 and $72 \mathrm{~h}$ post-infection (hpi), that r3MACV titers were lower $\left(2 \times 10^{4}\right.$ and $4 \times 10^{4}$ $\left.\mathrm{TCID}_{50} / \mathrm{ml}\right)$ than the MACV wt titers $\left(3 \times 10^{6}\right.$ and $1 \times 10^{7}$ $\mathrm{TCID}_{50} / \mathrm{ml}$ ) (Fig. 2a). We also noted that the r3MACV infected fewer Vero cells than the MACV wt (Fig. 2b). In conclusion, the r3MACV genomic modifications resulted in a decrease of the virus infectivity in Vero cells.

To test the genomic stability, an additional fifth passage of the r3MACV viral stock was produced using conditions previously described for the production [11]. Briefly, the supernatants from MOI 0,01 infected Vero cells were collected 3 days post infection and centrifuged $15 \mathrm{~min}, 1610 \mathrm{~g},+4{ }^{\circ} \mathrm{C}$ centrifuged before being passaged using these conditions. The viral stock and the late fifth passage were extracted (QIAmp Viral RNA Mini Kit, Qiagen) and sequenced. The experiments were done in triplicate. All results of r3MACV sequencing were similar. Sequencing revealed three changes between r3MACV and MACV wt in the L-segment. Two changes resulted in false sense mutation $(\mathrm{T} \rightarrow \mathrm{C})$ at position 1733 (leucine $\rightarrow$ proline) and at position 6598 (serine $\rightarrow$ proline) in the polymerase. We also identified a $35 \mathrm{nt}$ deletion in the intergenic region (on position 417 to 452 ). Sequencing revealed one change under one $\mathrm{S}$ segment of r3MACV: surprisingly, we found an 1124 nt deletion in the C-termini of the CHAPV GPC $\Delta 33$. These deletions were observed in the GP1 and GP2 sequences of the GPC, which are involved in the cellular attachment and entry of the virion $[9,12]$. Further experimentations will be needed to understand the consequences of these observations. However we can speculate that an excess of SSP-GP1 residual complex was expressed at the cell surface, moreover this truncated CHAPV GPC was likely not able to produce any infectious virions [12]. No genetic changes in the others genes were identified. In summary, r3MACV showed a moderate genetic stability after 5 passages, regarding the conservation of tri-segmented segments and the mutations inserted. However to support the safety of r3MACV, the deletion inside the CHAPV GPC $\triangle 33$ needed to be studied. The deletion of $35 \mathrm{nt}$ in the $\mathrm{L}$ segment is interesting. As shown by Golden and his collaborators, a variant of this viral strain, also having a deletion of $35 \mathrm{nt}$ but forward $8 \mathrm{nt}$ (position 409-443) in the intergenic region of $\mathrm{L}$ segment, is not pathogenic for guinea pigs [17]. These data suggested a confidence in the safety of r3MACV. Consequently, the CHAPV GPC $\triangle 33$ representation in Fig. 1b was not entirely present in the r3MACV virus.

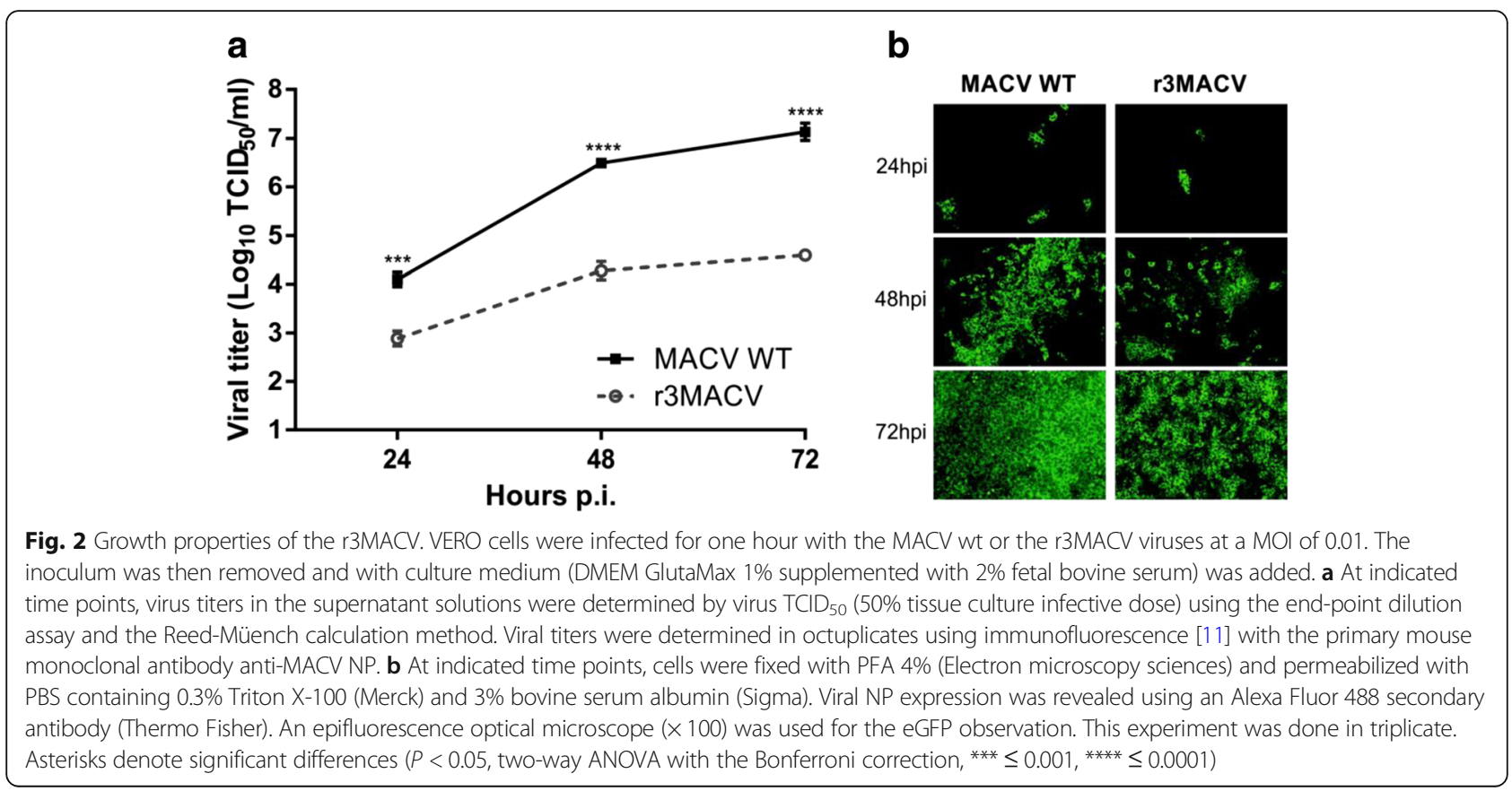


To assess that the r3MACV NP D380A mutation removed the NP wt inhibition of the host type-I IFN response, we compared the A549 cells IFN-1 $\beta$ production in different conditions (Fig. 3): infection at a MOI of 3 by MACV wt, r3MACV and Sendaï virus $(\mathrm{SeV})$ a known inducer of type-I IFN [18]. MACV wt was able to produce an infectious progeny (at $\left.3 \times 10^{6} \mathrm{TCID}_{50} / \mathrm{ml}\right)$ up to 24 hpi (Fig. 3a) whereas the infectious particles produced by r3MACV were essentially undetectable (Fig. 3a). SeV replication induced the production of IFN- $\beta$ at an average of $512 \mathrm{pg} / \mathrm{ml}$ (Fig. 3b), as previously reported. INF-1 $\beta$ was undetectable in cultures of MACV wtat 16 and 24 hpi (Fig. 3b) while r3MACV, similarly to $\mathrm{SeV}$, induced $527 \mathrm{pg} / \mathrm{ml}$ of IFN- $\beta$ (average) at $24 \mathrm{hpi}$ (Fig. 3b). We further confirmed this observation using RT-qPCR (Fig. 3c). Together, these findings demonstrated that $\mathrm{r} 3 \mathrm{MACV}$ is no longer capable to block the IFN production from infected cells.

In order to analyze the protective capability of r3MACV, Dunkin-Hartley guinea pigs (females, 200-250 g, Charles River) animals were inoculated by intraperitoneal injections in a $0.5 \mathrm{ml}$ PBS final volume in a biosafety level (BSL)-4 laboratory (Jean Merieux, Lyon, France). During all manipulation steps excluding weighing (3 times per week), guinea pigs were anesthetized by isoflurane $3 \%$ inhalation. Animals were humanly euthanized when end points were attained.

The MACV strain Carvallo pathogenicity has been demonstrated in guinea pigs (lethality 60\%) $[19,20]$. To determine the wt MACV lethal dose in this experiment, two groups of guinea pigs were inoculated using
$3 \times 10^{4} \mathrm{TCID}_{50}$ or $10^{6} \mathrm{TCID}_{50}$. In the two conditions, animals survived, without losing weight and did not develop any HHF symptoms (see Additional file 3). This result indicated that, in our hands, MACV wt was not lethal despite the high doses tested, also a recent observation by other researchers [21]. Therefore, we then chose the Espindola strain of JUNV, closely genetically related to MACV, which was shown to induce $100 \%$ mortality in guinea pig, following weight loss and hemorrhagic symptoms [22]. We also selected the Candid \#1 vaccine as comparison. Even if it requires 2 months following the vaccination to induce a protection against JUNV [14], we believed it would add meaningful information, as another live-attenuated virus. Following the JUNV challenge, we administered a low dose of r3MACV and Candid \#1. However, the r3MACV dose was 3 times lower than expected because the r3MACV production in VERO cells was low.

All control guinea pigs, receiving JUNV and PBS, developed severe symptoms before dying including high weight loss, dyspnea and decrease of tonus (Fig. 4a and b). Similar symptoms and outcomes were observed in all the non-protected animals in other group (Fig. 4a). Animals developing a fatal infection lost from 9 to $34 \%$ of their maximum weight (Fig. 4b, c and d). Surviving animals, either in the Candid \#1/JUNV group (one, 25\%) or in the r3MACV/JUNV group (two, 50\%), did not show any symptoms. The Candid \#1/JUNV group survivor, guinea pig \#1, lost up to $12.8 \%$ of its maximum weight from day 16 pi to day 21 pi, then regained $98.5 \%$ of its weight (Fig. 4c). The surviving guinea pigs \#1 and \#4 from
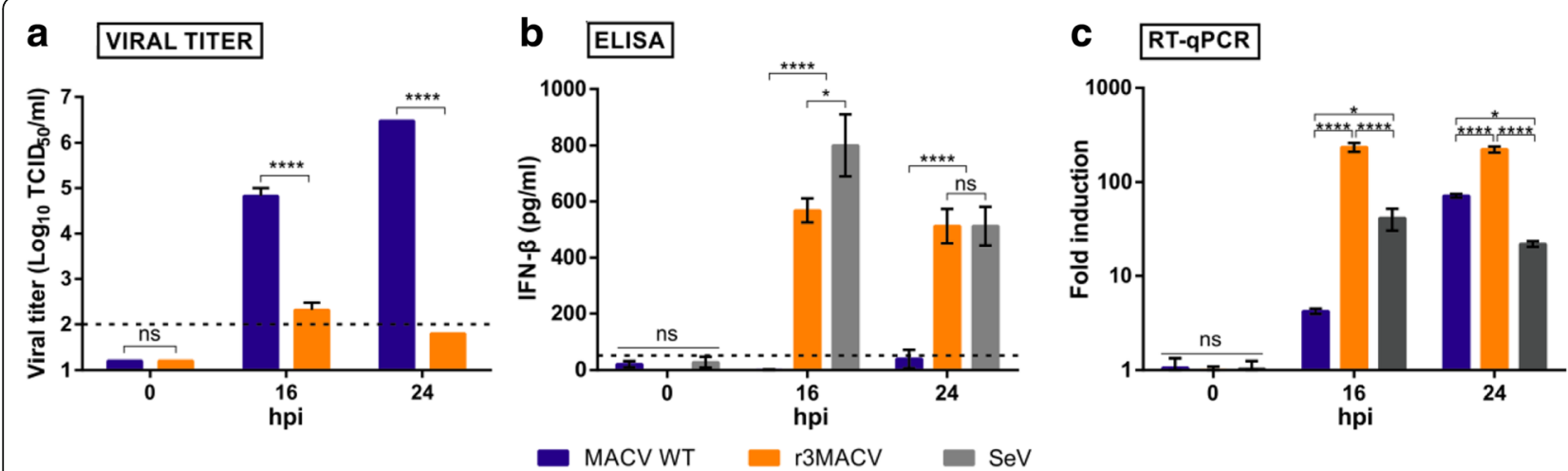

Fig. 3 IFN- $\beta$ production by A549 cells infected either with MACV wt, r3MACV or SeV. A549 cells were infected for one hour with the MACV wt, r3MACV or SeV viruses at a MOI of 3, then the inoculum was removed and fresh culture medium was added. At indicated time points, a virus titers in the supernatants were determined by virus $\mathrm{TCID}_{50}$, as described in Fig. 2 ; $\mathbf{b}$ production of IFN- $\beta$ was detected in supernatant solutions using the VeriKine Human IFN BETA ELISA Kit (PBL assay sciences); and c intracellular RNA was extracted using the QIAmp Viral RNA Mini kit (Qiagen). RT-qPCR were performed using the QuantiTect Probe RT-PCR kit (Qiagen) in a $30 \mu$ final volume with $5 \mu$ l of purified RNA and primers and probe at a final concentration of $400 \mathrm{nM}$ and $200 \mathrm{nM}$, respectively. The assay was carried out using a CFX96 model (Bio-Rad) with a cycling profile of $50{ }^{\circ} \mathrm{C}$ for $30 \mathrm{~min}, 95^{\circ} \mathrm{C}$ for $15 \mathrm{~min}$, and $40 \mathrm{cycles}$ at $94{ }^{\circ} \mathrm{C}$ for $15 \mathrm{~s}$ followed by $60^{\circ} \mathrm{C}$ for $1 \mathrm{~min}$. The primers and probes (available on request) were designed using the Eurofins Genomics' online tool (www.eurofinsgenomics.eu). Probes were $5^{\prime}$ - and 3'-labelled with the fluorescent reporter dye 6-carboxyfluorescein (FAM) and the Black Hole Quencher (BHQ-1), respectively. All procedures were carried out following manufacturers' instructions. This experiment was carried out in triplicate. Asterisks denote significant differences $(P<0.05$, two-way ANOVA with the Bonferroni correction, ns: no significate, ${ }^{*} \leq 0.05,{ }^{* * *} \leq 0.0001$ ) 

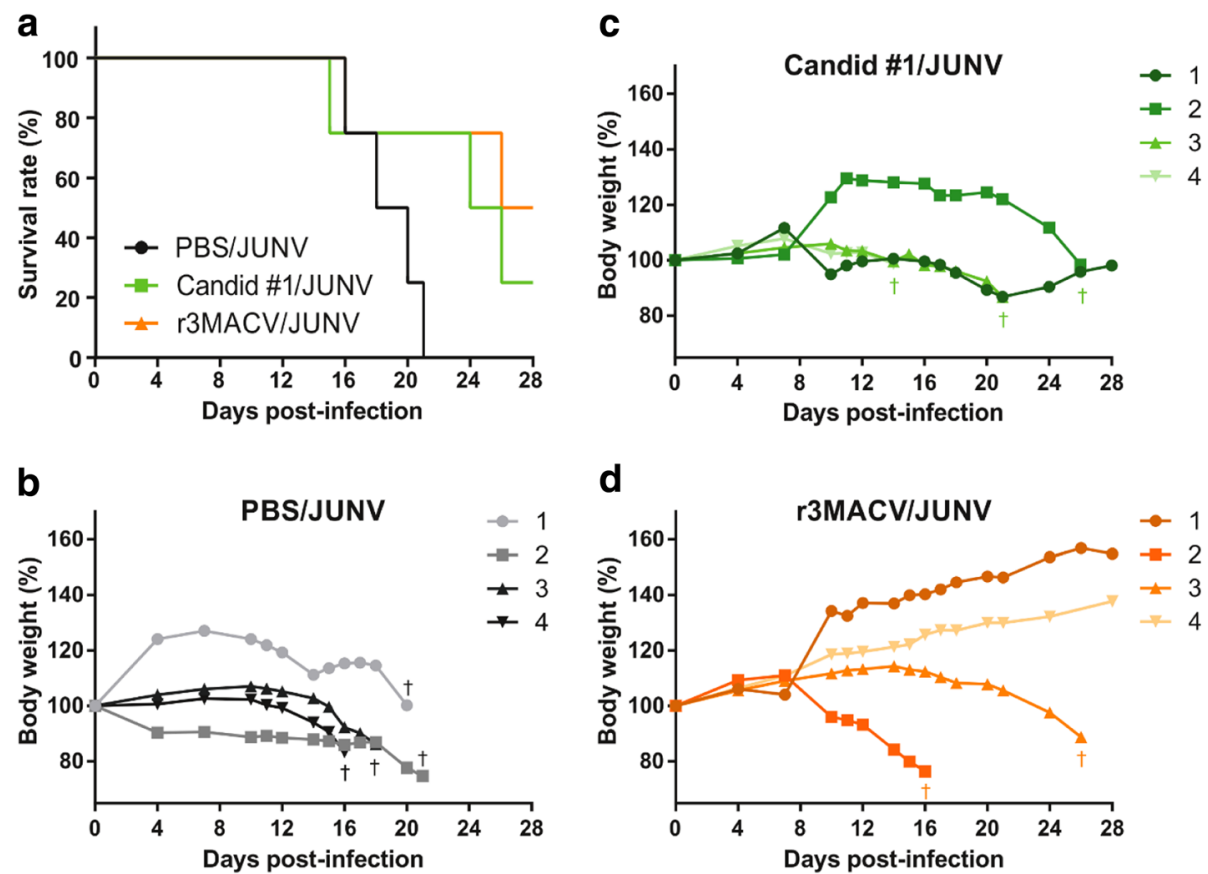

Fig. 4 Survival rate and weight changes of guinea pigs throughout the experiment. Three groups of Hartley guinea pigs $(n=4 /$ group) were immunized intraperitoneally in the right flank with $3 \times 10^{4} \mathrm{TCID}_{50}$ of Candid $\# 1,4 \times 10^{3} \mathrm{TCID}_{50}$ of $\mathrm{r}_{3 \mathrm{MACV}}$ or PBS (control). Simultaneously, in the left flank, a lethal challenge $\left(10^{4} \mathrm{TCID}_{50}\right.$ of JUNV strain Espindola) was injected intraperitoneally. a Their survival rate and b-d weight were observed for 28 days thereafter. The + indicated animals that either succumbed to the infection or were humanly euthanized before the end of the experiment

the r3MACV/JUNV group, regularly kept gaining weight, reaching at the end of the experiment 155 and $138 \%$ of their initial weight, respectively (Fig. $4 \mathrm{~d}$ ).

All guinea pigs which succumbed to the infection showed a detectable JUNV viral load in their serum at day 14, except guinea pig \#3 from the r3MACV/JUNV group, which died at $26 \mathrm{dpi}$ (Fig. 5a). We observed that when the guinea pig JUNV viremia was above $10^{2}$ $\mathrm{TCID}_{50} / \mathrm{ml}$ at day 14 , the guinea pig died between 14 and $21 \mathrm{dpi}$. None of the surviving animals exhibited any detectable viremia at day 14 . All dead animals had detectable virus titers in all tested organs except in the lung of animal \#2 of the Candid \#1/JUNV group, and in the kidney of animal \#2 of the r3MACV/JUNV group. All surviving animals did not exhibit any detectable viral titer in tested organs (Fig. 5b).

In conclusion, our study demonstrated that the r3MACV candidate was able to protect $50 \%$ of guinea pigs from a simultaneous lethal JUNV challenge. Surviving animal displayed neither clinical symptom nor detectable infectious virus in collected samples. This protection was higher as a therapeutic treatment than that provided by Candid \#1 (as a vaccine requiring two months for immunity with variable efficacy against different JUNV strains [14]). Moreover protection occurred at a 3-fold lower dose of r3MACVthan Candid \#1. Several factors may be considered: (i) as observed in vitro, the r3MACV might have induced a high level of type-I IFN production, resulting in the establishment of an antiviral state preventing the JUNV propagation, ultimately resulting in the control of the viral infection by the host immune system; (ii) the r3MACV might have competed with JUNV to enter cells, or induced the superinfection exclusion phenomenon [23], thereby limiting the ability of the pathogenic JUNV to infect animals; (iii) the r3MACV might have also elicited an adaptive immune response. These hypotheses will need to be tested in future experiments.

We could not test the attenuation of the r3MACV in a MACV wt lethal challenge as noted (Additional file 1) and it could be studied if such a successful animal model is identified. However, these studies suggest thatr3MACV will be a safe product because (i) the tri-segments modification of the mammarenavirus genome was associated with an attenuated phenotype [10], (ii) the NP D380A mutation was shown to block the inhibition of the IFN-1 $\beta$ production in A549 cells, (iii) the 2-log reduction ability of the $\mathrm{rMMCV}$ to grow in VERO cells, (iv) a MACV and JUNV recombination viruses could not be excluded but likely improbable [23].

Regarding the efficacy of Candid \#1 as a therapeutic vaccine, we showed that it was less protective than the r3MACV despite its closer antigenic relationship 


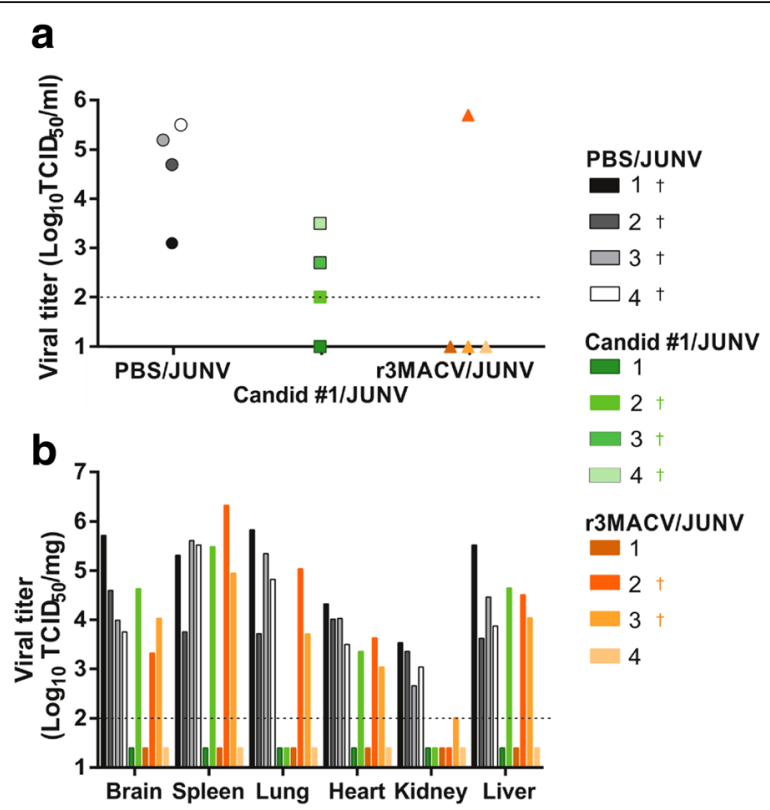

Fig. 5 JUNV viremia and viral titer in organs of quinea pig in the three conditions. a Blood samples were collected at 14 days post-infection in the retro-orbital sinus. Blood samples were centrifuged (1400 rpm for $3 \mathrm{~min}$ ) to collect sera. Virus titers were determined, with a limit of detection at $10^{2} \mathrm{TCID}_{50} / \mathrm{ml}$ (dotted line). b Following the euthanasia of animals, organs (brain, spleen, lung, left kidney, heart and liver) were collected and stored at $-80^{\circ} \mathrm{C}$. Organs of animals \#3 and \#4 of the C \#1/JUNV group were not collected because they were found dead with cadaveric rigidity. Collected organs were cut, weighted and crushed using Tissue Lyser in $400 \mu$ PBS (ThermoFisher Scientific). Supernatant solutions were harvested and used for virus titration, with a limit of detection at $10^{2} \mathrm{TCID}_{5} / \mathrm{mg}$ (dotted line). The † indicated animals that either succumbed to the infection or were humanly euthanized before the end of the experiment

with the virus used for the challenge. If the r3MACV fulfills the necessary requirements in term of safety and antigenic expression of GPC $\triangle 33$ inserted in each $\mathrm{S}$ segment, further experiments will be needed to address its potentially therapeutic and prophylactic protection against MACV and JUNV and ultimately against other South America arenaviruses responsible for HHF.

\section{Additional files}

Additional file 1: Impact of the mutation L166G on the vira polymerase main functions. BHK-21 cells were transfected with plasmids expressing the minigenome (MG), the MACV nucleoprotein (NP) and the wild-type AY619642 (L) or the 166G-mutated (E166G) polymerase in conditions previously published [11]. The plasmid expressing the MACV polymerase was replaced by a plasmid expressing the mCherry in the negative control. Three days post-transfection, the Gaussia Luciferase expression in the supernatant solution was evaluated using the Gaussia luciferase assay kit (New England Biolabs). Asterisks denote significant differences with control $(P<0.05$, one-way ANOVA with the Bonferroni correction, $\left.^{* * *} \leq 0.0001\right)$. (PDF $313 \mathrm{~kb}$ )

Additional file 2: Genomic sequences of r3MACV. (PDF $336 \mathrm{~kb}$ )
Additional file 3: Survival rate and body weight of guinea pig inoculated with two doses of wt MACV. Four or five guinea pigs were infected intraperitoneally with $3 \times 10^{4} \mathrm{TCID}_{50}$ (low dose) or $10^{6} \mathrm{TCID}_{50}$ (high dose) of wt MACV, respectively. The animals were observed for 24 days thereafter. (A) Their survival rate and (B) their weight were monitored. Animals infected with the high dose were heavier $(515-610 \mathrm{~g})$ than the ones used for the low dose experiment (250-300 g), explaining the differences of weight evolution between the two groups. (PDF $375 \mathrm{~kb}$ )

\section{Abbreviations}

BHF: Bolivian Hemorrhagic Fever; BSL: Biosafety Level; CHAPV: Chapare virus; GPC: Glycoprotein complex; GTOV: Guanarito virus; HHF: Human

Hemorrhagic Fever; hpi: Hours post-infection; IFN: Interferon; JUNV: Junín virus; LCMV: Lymphocytic choriomeningitis virus; MACV: Machupo virus; MOI: Multiplicity of infection; NHPs: Non-human primates; NP: Nucleoprotein; SABV: Sabiá virus; wt: Wild-type

\section{Acknowledgements}

The authors thanked Dr. Sébastien Emonet for his scientific advice and involvement in design of this study. We are grateful to Fanny Jarjaval, Isabelle Drouet, Lisa Lartigau and Dr. Marie Moroso for their technical support. We thank Pasteur Institute (UBIVE) for providing us with the Sendai virus. We also thank P4 in vivo team for the realization of the experimental study in the BSL4 Inserm-Jean Mérieux. We are indebted to Dr. Marti Jett for revising the manuscript.

\section{Funding}

This work was supported by a DGA-MRIS scholarship to A.D.Z., n007/2014/ DGA. The funder had no role in study design, data collection and analysis, or preparation of the manuscript, but had role in decision to publish. The research was partially funded by the Direction Générale de l'Armement and the Service de Santé des Armées, Biomedef PDH-2-NRBC-3-B2-304. The funder had no role in study design, data collection and analysis, decision to publish, or preparation of the manuscript.

\section{Avaibility of data and materials}

All data generated or analyzed during this study are included in this published article [and its supplementary information files].

\section{Authors' contributions}

ADZ, CHH and CNP designed this study and analyzed the results. ADZ performed the experiments. ADZ drafted the manuscript. $\mathrm{CHH}$ and CNP revised the manuscript critically. All of the authors read and approved the final version of this manuscript.

\section{Ethics approval}

All experiment in guinea pigs were approved by an external ethic committee named CECCAPP NCEO15 (Permit number APAFIS\#8168-201,611,241 017566 V2) according to the Directive 2010/63/EU in Europe.

\section{Competing interests}

The authors declare that they have no competing interests.

\section{Publisher's Note}

Springer Nature remains neutral with regard to jurisdictional claims in published maps and institutional affiliations.

\section{Author details}

${ }^{1}$ Fab'entech, 24 rue Jean Baldassini Bat B 69007, Lyon, France. ${ }^{2}$ Unité de virologie, Institut de Recherche Biomédicale des Armées, 1 place Valérie André, 91220 Brétigny-sur-Orge, France. ${ }^{3}$ UMR 190, Faculté de Médecine-Timone, 27 boulevard Jean-Moulin, 13385 Marseille, France.

Received: 16 November 2017 Accepted: 1 June 2018

Published online: 07 June 2018

\section{References}

1. Johnson KM, Mackenzie RB, Webb PA, Kuns ML. Chronic infection of rodents by Machupo virus. Science. 1965;150(3703):1618-9. 
2. Aguilar PV, Camargo W, Vargas J, Guevara C, Roca Y, Felices V, LagunaTorres VA, Tesh R, Ksiazek TG, Kochel TJ. Reemergence of Bolivian hemorrhagic fever, 2007-2008. Emerg Infect Dis. 2009;15(9):1526-8.

3. Enria DA, Briggiler AM, Sánchez Z. Treatment of argentine hemorrhagic fever. Antivir Res. 2008;78(1):132-9.

4. Radoshitzky SR, Bào Y, Buchmeier MJ, Charrel RN, Clawson AN, Clegg CS, DeRisi JL, Emonet S, Gonzalez JP, Kuhn JH, Lukashevich IS, Peters CJ, Romanowski V, Salvato MS, Stenglein MD, de la Torre JC. Past, present, and future of arenavirus taxonomy. Arch Virol. 2015;160(7):1851-74.

5. de Manzione N, Salas RA, Paredes H, Godoy O, Rojas L, Araoz F, Fulhorst CF, Ksiazek TG, Mills JN, Ellis BA, Peters CJ, Tesh RB. Venezuelan hemorrhagic fever: clinical and epidemiological studies of 165 cases. Clin Infect Dis. 1998; 26(2):308-13.

6. Delgado S, Erickson BR, Agudo R, Blair PJ, Vallejo E, Albariño CG, Vargas J, Comer JA, Rollin PE, Ksiazek TG, Olson JG, Nichol ST. Chapare virus, a newly discovered arenavirus isolated from a fatal hemorrhagic fever case in Bolivia. PLoS Pathog. 2008:4(4):1-6.

7. Coimbra TLM, Coimbra M, Nassar ES, Burattini MN, de Souza LT, Ferreira I, Rocco IM, da Rosa AP, Vasconcelos PF, Pinheiro FP, et al. New arenavirus isolated in Brazil. Lancet. 1994;343(8894):391-2.

8. Eddy GA, Wagner FS, Scott SK, Mahlandt BJ. Protection of monkeys against Machupo virus by the passive administration of Bolivian hemorrhagic fever immunoglobulin (human origin). Bull World Health Organ. 1975;52(4-6):723-7.

9. Buchmeier M, de la Torre JC. Arenaviridae: the viruses and their replication. In: Knipe DM, Howley PM, editors. Fields Virology, vol. 2. 5th ed. Philadelphia, PA: Lippincott-Williams \& Wilkins Publishers; 2007. p. 1792-827.

10. Emonet SF, Garidou L, McGavern DB, de la Torre JC. Generation of recombinant lymphocytic choriomeningitis viruses with trisegmented genomes stably expressing two additional genes of interest. Proc Natl Acad Sci U S A. 2009;106(9):3473-8.

11. Emonet SF, Seregin AV, Yun NE, Poussard AL, Walker AG, de la Torre JC, Paessler S. Rescue from cloned cDNAs and in vivo characterization of recombinant pathogenic Romero and live-attenuated candid \#1 strains of Junin virus, the causative agent of argentine hemorrhagic fever disease. J Virol. 2011;85(4):1473-83.

12. Kunz S, Edelmann KH, de la Torre JC, Gorney R, Oldstone MB. Mechanisms for lymphocytic choriomeningitis virus glycoprotein cleavage, transport, and incorporation into virions. Virology. 2003;314(1):168-78.

13. Martínez-Sobrido L, Emonet S, Giannakas P, Cubitt B, García-Sastre A, de la Torre JC. Identification of amino acid residues critical for the anti-interferon activity of the nucleoprotein of the prototypic arenavirus lymphocytic choriomeningitis virus. J Virol. 2009;83(21):11330-40.

14. Enria DA, Barrera Oro JG. Junin virus vaccines. Curr Top Microbiol Immunol. 2002;263:239-61.

15. Lee KJ, Novella IS, Teng MN, Oldstone MB, de La Torre JC. NP and L proteins of lymphocytic choriomeningitis virus (LCMV) are sufficient for efficient transcription and replication of LCMV genomic RNA analogs. J Virol. 2000;74(8):3470-7.

16. Flatz $L$, Bergthaler $A$, de la Torre JC, Pinschewer DD. Recovery of an arenavirus entirely from RNA polymerase I/II-driven CDNA. Proc Natl Acad Sci U S A. 2006;103(12):4663-8.

17. Golden JW, Beitzel B, Ladner J, Mucker EM, Kwilas SA, Palacios G, Hooper JW. An attenuated Machupo virus with a disrupted L-segment intergenic region protects Guinea pigs against lethal Guanarito virus infection. Sci Rep. 2017;7(1):4679.

18. Strahle L, Garcin D, Kolakofsky D. Sendai virus defective-interfering genomes and the activation of interferon-beta. Virology. 2006;351(1):101-11.

19. Peters CJ, Kuehne RW, Mercado RR, Le Bow RH, Spertzel RO, Webb PA. Hemorrhagic fever in Cochabamba, Bolivia, 1971. Am J Epidemiol. 1974; 99(6):425-33.

20. Golden JW, Hammerbeck CD, Mucker EM, Brocato RL. Animal models for the study of rodent-borne hemorrhagic fever viruses: arenaviruses and hantaviruses. Biomed Res Int. 2015;2015:793257.

21. Bell TM, Bunton TE, Shaia Cl, Raymond JW, Honnold SP, Donnelly GC, Shamblin JD, Wilkinson ER, Cashman KA. Pathogenesis of Bolivian hemorrhagic fever in Guinea pigs. Vet Pathol. 2016;53(1):190-9.

22. Peters $C J$, Jahrling PB, Liu CT, Kenyon RH, McKee KT Jr, Barrera Oro JG. Experimental studies of arenaviral hemorrhagic fevers. Curr Top Microbiol Immunol. 1987:134:5-68

23. Ellenberg $P$, Linero FN, Scolaro LA. Superinfection exclusion in BHK-21 cells persistently infected with Junín virus. J Gen Virol. 2007:88(Pt 10):2730-9.

\section{Ready to submit your research? Choose BMC and benefit from:}

- fast, convenient online submission

- thorough peer review by experienced researchers in your field

- rapid publication on acceptance

- support for research data, including large and complex data types

- gold Open Access which fosters wider collaboration and increased citations

- maximum visibility for your research: over $100 \mathrm{M}$ website views per year

At BMC, research is always in progress.

Learn more biomedcentral.com/submissions 